\title{
Prevalence of feline haemoplasma in cats in Denmark
}

\author{
Maja Benedicte Rosenqvist ${ }^{1,2+}$, Ann-Katrine Helene Meilstrup ${ }^{1,2+}$, Jesper Larsen ${ }^{1,3}$, John Elmerdahl Olsen ${ }^{1}$, \\ Asger Lundorff Jensen ${ }^{2}$ and Line Elnif Thomsen ${ }^{1 *}$
}

\begin{abstract}
Background: Infections with the three feline haemotropic mycoplasmas Mycoplasma haemofelis, Candidatus Mycoplasma haemominutum and Candidatus Mycoplasma turicensis cause feline infectious anemia. The purpose of this study was to investigate the prevalence of carriage of feline haemoplasma in Danish cats in different age groups. The presence was detected by a conventional polymerase chain reaction (PCR) assay on blood samples as well as by realtime PCR (RT-PCR).

Results: The study revealed a prevalence of 14.9\% Candidatus Mycoplasma haemominutum positive cats and 1.5\% Mycoplasma haemofelis positive cats. No cats were found positive for Candidatus Mycoplasma turicensis. The results showed a statistically significant higher prevalence in older (>8 years) cats compared to younger cats and a higher prevalence among domestic cats compared to purebred cats. As part of this study, we developed a cloning strategy to obtain Danish positive controls of haemoplasma 16S rRNA.

Conclusion: From convenience-sampled cats in Denmark, we found that $16.4 \%$ were carriers of feline haemotropic mycoplasmas. Haemoplasma was mostly found in older and domestic cats. The prevalence found in Denmark is similar to that found in several other European countries.
\end{abstract}

Keywords: Feline haemoplasma, Prevalence, Real-time PCR, Conventional PCR, Risk factors

\section{Background}

Feline haemotropic mycoplasmas (haemoplasmas) are small epierythrocytic Gram-negative bacteria, which can only survive by parasitism of erythrocytes [1] and which cause feline infectious anemia. The feline haemoplasma group consists of at least three species, Mycoplasma haemofelis (Mhf), Candidatus Mycoplasma haemominutum $(\mathrm{CMhm})$ and Candidatus Mycoplasma turicensis (CMt) [1-3]. Infections with these three species differ in severity with Mhf inducing the most severe symptoms such as anorexia, depression, fever and anemia; whereas the CMhm and CMt infected cats rarely show any clinical signs [4-6]. CMhm usually require co-infection with

\footnotetext{
*Correspondence: leth@sund.ku.dk

${ }^{\dagger}$ Maja Benedicte Rosenqvist and Ann-Katrine Helene Meilstrup contributed equally to this work

${ }^{1}$ Department of Veterinary Disease Biology, Faculty of Health and Medical Sciences, University of Copenhagen, Stigboejlen 4, 1870 Frederiksberg C, Denmark

Full list of author information is available at the end of the article
}

another haemoplasma or immune-compromised conditions to cause disease and the pathogenic potential of CMt most likely also depends on cofactors [7-9].

Feline haemoplasmas are widely distributed in pet cats throughout the world, although the prevalence varies geographically [9-13]. These variations may be due to differences in climate as studies have shown a correlation between haemoplasma prevalence and warmer climate [9-11, 14]. The higher prevalence is suggested to be due to presence of a higher number of blood-sucking arthropods, which are suspected to be vectors for haemoplasmas $[12,15]$. The risk of infection has also been associated with several other factors, such as age, gender and breed [10-12].

Haemoplasmas have never been cultured in vitro and identification is usually based on cytological examination of blood smears [6]. However, this method has proven inefficient due to low sensitivity and specificity resulting in false negative results. Development of PCR based 
methods has increased the sensitivity of identification of the organisms in blood samples [9, 12, 16-18]. Feline infectious anemia was initially reported in Denmark in 1974, but the prevalence is unknown [18]. In this study, we used PCR methods to determine the prevalence of feline haemoplasmas in the blood of convenience samples of cats from Denmark and evaluated possible associations to breed, age, gender and symptoms.

\section{Methods}

\section{Collection of blood samples and clinical information}

Seventy-three blood samples, representing blood from 67 cats sampled during 2007-2008 were used. Forty-six of the samples had been collected from patients at the University Hospital for Companion Animals, Faculty of Health and Medical Sciences, University of Copenhagen. The remaining 27 blood samples were collected at veterinary clinics throughout Denmark. The blood samples were collected from both diseased cats and cats attending the clinics for routine health checks. From four of the cats from the University Hospital we had samples in duplicate or triplicate. These samples were used as controls for the PCR reaction to confirm that we obtained identical results on repeated testing. For 62 cats, information on age, breed and gender was available. Anamnesis and clinical findings were available for all cats admitted to the University Hospital and from ten samples from the other clinics. The blood samples were obtained by venal puncture and stored in $2.5 \mathrm{ml}$ EDTA-vials at $-80{ }^{\circ} \mathrm{C}$. Blood samples obtained from the University Hospital were all subjected to haematological examination using the ADVIA 120 haematology system including erythrocyte count, haemoglobin-, haematocrit- and mean cell volume $(\mathrm{MCV})$ values.

\section{DNA extraction}

Extraction of total DNA from the blood samples was done using the DNeasy ${ }^{\circledR}$ Blood and Tissue kit (Qiagen, Denmark). $100 \mu \mathrm{l}$ EDTA blood and $100 \mu \mathrm{l}$ PBS was used for each DNA purification.

\section{PCR amplification and cloning of 16S rRNA from haemoplasma}

Conventional PCR: $0.8 \mu \mathrm{M}$ of each primer M-forward: $5^{\prime}$ ACGAAAGTCTGATGGAGCAATA $3^{\prime}$ and M-reverse: $5^{\prime}$ ACGCCCAATAAATCCGRATAAT 3' [5]. PCR was performed using the Thermo Hybaid PCR express machine using the following conditions, Segment $1: 94{ }^{\circ} \mathrm{C} 1 \mathrm{~min}$, Segment 2: $94{ }^{\circ} \mathrm{C} 1 \mathrm{~min}, 60{ }^{\circ} \mathrm{C} 1 \mathrm{~min}, 72{ }^{\circ} \mathrm{C} 30 \mathrm{~min}$ at 45 cycles and Segment 3: $72^{\circ} \mathrm{C}$ for $10 \mathrm{~min}$.

RT-PCR: $0.3 \mu \mathrm{M}$ of the HPLC purified primers, M-forward: $5^{\prime}$ ACGAAAGTCTGATGGAGCAATA $3^{\prime}$ and M-reverse: 5' ACGCCCAATAAATCCGRATAAT 3' [5].
RT-PCR was performed using the $\mathrm{Mx} 30000 \mathrm{P}^{\circledR}$ and Brilliant ${ }^{\circledR}$ SYBR Green QPCR master mix (Stratagene, California, USA) essentially as described by the manufacturer. The RT-PCR reaction was run under the following conditions: Segment 1: Initial denaturation $95{ }^{\circ} \mathrm{C} 10 \mathrm{~min}$, Segment 2: $95{ }^{\circ} \mathrm{C} 30 \mathrm{~s}, 60{ }^{\circ} \mathrm{C} 1 \mathrm{~min}, 72{ }^{\circ} \mathrm{C} 30 \mathrm{~s}$, for 40 cycles, Segment 3: $95^{\circ} \mathrm{C} 1 \mathrm{~min}$, ramp down to $55^{\circ} \mathrm{C}$ and ramp up from 55 to $95{ }^{\circ} \mathrm{C}$. Dissociation temperatures were $77.4{ }^{\circ} \mathrm{C}$ for $\mathrm{CMhm}, 78.0{ }^{\circ} \mathrm{C}$ for Mhf and $79.5^{\circ} \mathrm{C}$ for CMt. Primers for both conventional and RT-PCR amplify products of $193 \mathrm{bp}$ for CMhm and $170 \mathrm{bp}$ for both Mhf and CMt.

DNA from Mhf, CMhm and CMt was used as positive controls for the PCR methods [19]. The samples were used as positive controls in the initial analysis to confirm that the used PCR and RT-PCR conditions could produce the expected PCR products. After confirmation, the 16S rRNA PCR fragments from the first haemoplasmapositive blood samples ( $\mathrm{Mhf}$ and $\mathrm{CMhm}$ ) were used and cloned using the TOPO TA Cloning ${ }^{\circledR}$ Kit as described by the manufacturer (Invitrogen, Denmark). Since we found no CMt positive cats, we also cloned the CMt $16 \mathrm{~S}$ rRNA. All cloned fragments were sequenced, confirming that the DNA was of the expected sequence. Plasmid purification was done using the QIAprep ${ }^{\circledR}$ spin miniprep kit from Qiagen. Sequencing of the cloned samples was done using $0.8 \mu \mathrm{M}$ of each of the primers M13-forward: $5^{\prime}$ GTAAAACGACGGCCAGT $3^{\prime}$ and M13-reverse: $5^{\prime}$ AACAGCTATGACCATG $3^{\prime}$. These plasmids were used as positive controls in the PCR and RT-PCR analysis of the blood samples using the M-forward and M-reverse primers as described under the two PCR methods.

\section{DNA sequencing}

DNA sequencing of PCR products was performed on all positive samples (DNA Technology A/S). The sequence identity was confirmed by alignment to identity to GenBank accession number EU170604 and EU839985 (Mhf), EU839977 (CMt) and to EU839978 (CMhm).

\section{Statistical analysis}

Statistical analysis on the available information on gender, age and breed was done using GraphPad Prism 5 (https://www.graphpad.com). Fischer's exact test was used to test for association between presence of Mhf/ $\mathrm{CMhm}$ and age, gender and breed. Mann-Whitney U-test was used to test for significant differences in the haematological variables.

\section{Results}

Prevalence of carriage of feline haemoplasma species

Blood samples collected from 67 Danish cats were investigated to estimate the prevalence of carriage of the three haemoplasmas, CMhm, Mhf and CMt. For each cat, 
information about breed, gender, age and symptoms/ diagnosis were obtained when possible (Additional file 1: Table S1). The haemoplasmas are non-culturable in vitro and accurate diagnosis is currently reliant on detection of bacterial DNA using PCR assays [20]. Eleven animals were found to be positive among the 67 cats tested (Table 1). Ten cats were positive using both conventional and RT-PCR while one additional cat was positive by RTPCR only. Carriage of multiple haemoplasma species was not detected. From four PCR negative cats [cats 4, 5, 10, 15 (Additional file 1: Table S1)], more than one sample was analyzed [samples 51, 11, 38, 29, 41 and 55 (Additional file 1: Table S1)], and all samples yielded the same negative result upon repeated testing.

\section{Confirmation of $\mathrm{PCR}$ positive samples}

Based on fragment sizes, 10 positive samples were judged as harboring $\mathrm{CMhm}$, while one fragment corresponded to Mhf/CMt. Since the PCR products of Mhf and CMt positive cats are of equal size, all PCR products were sequenced to confirm the results and discriminate between these two species. The sequencing revealed that the $10 \mathrm{CMhm}$ positive samples indeed contained DNA from $C M h m$. Seven had $100 \%$ identity (E value: $3 \mathrm{e}^{-74}$ ) to GenBank accession number EU170604 and three had 97-100\% identity (E value: $3 \mathrm{e}^{-69}$ ) to EU839985. The one sample identified as $\mathrm{Mhf} / \mathrm{CMt}$ showed $98 \%$ identity (E value: $3 \mathrm{e}^{-58}$ ) to Mhf EU839978. We found no samples with homology to CMt among the Mhf/CMt positive samples, corresponding to $0 \%$ prevalence with a confidence interval between 0 and $4.7 \%$. Control DNA from CMt did result in the expected sequence of the PCR fragment, showing that the primers did amplify the CMt DNA.

\section{Association between presence of haemoplasma and clinical signs}

The statistical tests for significant differences in haematological values in cats being CMhm positive/negative showed no significant differences $(\mathrm{P}>0.5)$. One cat (Additional file 1: Table S1, no. 73) which was positive for Mhf, suffered from dehydration, weight loss and anorexia and the haematology of this cat showed abnormal values.
Haemoglobin, haematocrit and total erythrocyte counts were decreased, whereas MCV was increased compared to normal reference interval (Table 2). The blood smear showed several epierythrocytic organisms on every erythrocyte, anisocytosis, polychromasia and regeneration with reticulocytes and metarubricytes. The smear also contained lymphoblasts.

\section{Association between carriage of haemoplasma and age, breed and sex}

The univariable analyses for risk factors showed significantly higher carriage frequency in cats $\geq 8$ years old compared to younger cats $(\mathrm{P}<0.05)$, and a higher prevalence among domestic cats compared to purebred cats $(\mathrm{P}<0.05)$. One of the cats (Additional file 1: Table S1, no. 42) was diagnosed as feline immunodeficiency virus (FIV) positive and was also infected with CMhm. Among males, 23.5\% (8/34) carried haemoplasma species, whereas only $9.4 \%(3 / 32)$ of the females were positive. Although, prevalence in males seemed higher than in females, the difference was not statistically significant $(\mathrm{P}>0.05)$.

\section{Discussion}

The current study provides the first prevalence estimate of carriage of feline haemoplasma species in Denmark. The prevalence of CMhm and Mhf infections was 14.9 and $1.5 \%$ respectively when using RT-PCR. One sample, being positive by RT-PCR, was not identified as CMhm when using conventional PCR. Previous studies have shown a similar prevalence in other European countries $[9,19,20]$. The study was performed by the use of a convenience-sampled cat population. The cats included were examined by a veterinarian and represented both diseased cats and cats attending the clinic for routine health checks. This corresponds to the approach used in other studies $[11,13]$.

The most frequent haemoplasma found in the Danish cats was $\mathrm{CMhm}$. Feline CMhm infections are often subclinical with only minor haematological changes $[17,22$, 23] and in accordance with this, we found no significant difference in haematological values in cats being $\mathrm{CMhm}$ positive or negative.

Table 1 Haemoplasma prevalence in blood from 67 cats in Denmark determined by PCR and RT-PCR

\begin{tabular}{lcccccc}
\hline Species & Positive by PCR & Prevalence PCR (\%) & $\mathbf{9 5 \% ~ C l}$ & Positive by RT-PCR & Prevalence RT-PCR (\%) & 95\% Cl \\
\hline Mhf & 1 & 1.5 & $0-4.4$ & 1 & 1.5 & $0-4.4$ \\
CMhm & 9 & 13.4 & $5.2-21.6$ & 10 & 14.9 & $6.4-23.4$ \\
CMt & 0 & & & 0 & 16.4 & $7.5-25.3$ \\
Total & 10 & 14.9 & $6.4-23.4$ & 11 & \\
\hline
\end{tabular}

Mhf Mycoplasma haemofelis, CMhm Candidatus Mycoplasma haemominutum, CMt Candidatus Mycoplasma turicensis, Cl confidence interval 
Table 2 Haematology results for Mhf positive cat no. 73

\begin{tabular}{lcc}
\hline Parameter & Value & Reference interval \\
\hline Total erythrocyte count (billion/L) & 1.49 & $5-10$ \\
Haemoglobin (mmol/L) & 2.00 & $5-9.3$ \\
Haematocrit (L/L) & 0.09 & $0.24-0.45$ \\
Mean cell volume (MCV) (fL) & 60.4 & $40-57$ \\
\hline
\end{tabular}

This higher prevalence of CMhm carriage compared to the other haemoplasma species could be due to the lack of symptoms in these cats thus possibly allowing propagation and spread of the agent. Transmission between cats has been suggested, but not definitely demonstrated, to occur through biting wounds and from mother to offspring. Also haemoplasma DNA has been detected in the cat flea Ctenocephalides felis and ticks [12, 24]. Furthermore, these asymptomatic cats may impose a risk with the increased use of feline blood for transfusion. The only case of Mhf infection showed signs of haemoplasmosis with major haematological changes with a marked decreased erythrocyte count, and abnormal haemoglobin and haematocrit values. Furthermore the cat had an increased reticulocyte count and presence of nucleated erythrocytes. These findings supports the current view that Mhf is the most pathogenic species, and the one most often found associated with clinical disease [25].

We did not detect any CMt in the cats sampled in this study. Other prevalence studies have previously shown that CMt seems to be the least prevalent haemoplasma in Europe, ranging from 0 to $2.3 \%$ [12, 21, 26, 27]. In these studies, the sample sizes were between 3 to 20 times larger than our number of cats. A larger sample size could therefore be needed in order to conclude whether CMt is present in Denmark.

The significance of a positive PCR result should always be compared with clinical signs, pathological findings, haematological features and possible concurrent or complicating diseases. More severe clinical signs have occurred in cats experimentally dual-infected with $\mathrm{Mhf}$ and $\mathrm{CMhm}$ than in cats with mono-infection with either of the species and in cats spontaneously infected with both Mhf and CMhm [6, 28]. Based on PCR and sequencing of the PCR products, we found no samples with more than one haemoplasma species. The only CMhm positive cat in our study was diagnosed as FIV positive. The risk of infections with haemoplasma has previously been associated with concurrent diseases such as feline leukemia virus (FeLV) and FIV. Cats co-infected with FeLV develop a more severe anaemia than cats only infected with CMhm and FIV infection was shown to be associated with an increased risk of co-infection with CMhm and Mhf [7, 14, 29].

The risk of infection with feline haemoplasmas has been associated with different factors, such as age, gender, breed, environment, flea infestation and concurrent diseases [10-12, 15, 21]. Our results showed a statistical association between age and carriage of haemoplasma species, with an increased prevalence in older cats. These finding are consistent with findings in previous studies [12, 21, 26]. An association between risk of infection and increasing age may simply reflect a cumulative risk of exposure, since complete clearance of the organism once a cat has become infected has not been demonstrated. We also found higher prevalence of haemoplasma in domestic cats compared to purebred cats. Purebred cats are often held indoor, whereas domestic cats more often allowed being outdoor, where risk of exposure is higher.

\section{Conclusions}

This is the first study to investigate carriage of feline haemoplasma among Danish cats. The study showed a prevalence of $16.4 \%$ with $\mathrm{CMhm}$ as the most prevalent species. Age and breed was significantly associated with haemoplasma carriage. These results are in accordance with similar studies from other parts of Europe, USA and Japan.

\section{Additional file}

Additional file 1: Table S1. Sample characteristics of 67 cats, including age, gender, breed, symptoms and haemoplasma status.

\section{Authors' contributions}

$A L J$ and LET planned the study. MBR, AHM, JL performed all experiments and laboratory analysis. JEO, AHM and MBR did the statistics. LET wrote the manuscript. JEO, AL and JL commented on the manuscript. All authors read and approved the final manuscript.

\section{Author details}

${ }^{1}$ Department of Veterinary Disease Biology, Faculty of Health and Medical Sciences, University of Copenhagen, Stigboejlen 4, 1870 Frederiksberg C, Denmark. ${ }^{2}$ Department of Veterinary Clinical and Animal Sciences, Faculty of Health and Medical Sciences, University of Copenhagen, Dyrlaegevej 16, 1870 Frederiksberg C, Denmark. ${ }^{3}$ Statens Serum Institut, Artillerivej 5 , 2300 Copenhagen S, Denmark.

\section{Acknowledgements}

We thank Séverine Tasker and lain R. Peters, University of Bristol, for providing control DNA from Mhf, CMt and CMhm.

\section{Competing interests}

The authors declare that they have no competing interests.

Received: 6 April 2016 Accepted: 1 November 2016

Published online: 10 November 2016

\section{References}

1. Neimark H, Johansson KE, Rikihisa Y, Tully JG. Proposal to transfer some members of the genera Haemobartonella and Eperythrozoon to the genus Mycoplasma with descriptions of 'Candidatus Mycoplasma haemofelis,' 'Candidatus Mycoplasma haemomuris,' 'Candidatus Mycoplasma haemosuis' and 'Candidatus Mycoplasma wenyonii'. Int J Syst Evol Microbiol. 2001;51:891-9. 
2. Foley JE, Pedersen NC. Candidatus Mycoplasma haemominutum, a low-virulence epierythrocytic parasite of cats. Int J Syst Evol Microbiol. 2001:51:815-7.

3. Willi B, Boretti FS, Cattori V, Tasker S, Meli ML, Reusch C, et al. Identification, molecular characterisation and experimental transmission of a new hemoplasma isolate from a cat with hemolytic anaemia in Switzerland. J Clin Microbiol. 2005:43:2581-5.

4. Foley JE, Harrus S, Poland A, Chomel B, Pedersen NC. Molecular, clinical, and pathologic comparison of two distinct strains of Haemobartonella felis in domestic cats. Am J Vet Res. 1998;59:1581-8.

5. Jensen WA, Lappin MR, Kamkar S, Reagan WJ. Use of a polymerase chain reaction assay to detect and differentiate two strains of Haemobartonella felis in naturally infected cats. Am J Vet Res. 2001;62:604-8.

6. Westfall DS, Jensen WA, Reagan WJ, Radecki SV, Lappin MR. Inoculation of two genotypes of Haemobartonella felis (California and Ohio variants) to induce infection in cats and the response to treatment with azithromycin. Am J Vet Res. 2001;62:687-91.

7. George JW, Rideout BA, Griffey SM, Pedersen NC. Effect of preexisting FeLV infection or FeLV and feline immunodeficiency virus coinfection on pathogenicity of the small variant of Haemobartonella felis in cats. Am J Vet Res. 2002;63:1172-8.

8. Tasker S, Caney SMA, Day MJ, Dean RS, Helps CR, Knowles TG, et al. Effect of chronic FIV infection, and efficacy of marbofloxacin treatment, on 'Candidatus Mycoplasma haemominutum' infection. Microbes Infect. 2006;8:653-61.

9. Willi B, Tasker S, Boretti FS, Doherr MG, Cattori V, Meli ML, et al. Phylogenetic analysis of "Candidatus Mycoplasma turicensis" isolates from pet cats in the United Kingdom, Australia, and South Africa, with analysis of risk factors for infection. J Clin Microbiol. 2006:44:4430-5.

10. Lobetti RG, Tasker S. Diagnosis of feline haemoplasma infection using a real-time PCR assay. J S Afr Vet Assoc. 2004;75:94-9.

11. Tasker S, Braddock JA, Baral R, Helps CR, Day MJ, Gruffydd-Jones TJ, et al. Diagnosis of feline haemoplasma infection in Australian cats using a realtime PCR assay. J Feline Med Surg. 2004;6:345-54.

12. Willi B, Boretti FS, Baumgartner C, Tasker S, Wenger B, Cattori V, et al Prevalence, risk factor analysis, and follow-up of infections caused by three feline hemoplasma species in cats in Switzerland. J Clin Microbiol. 2006;44:961-9.

13. Jenkins KS, Dittmer KE, Marshall JC, Tasker S. Prevalence and risk factor analysis of feline haemoplasma infection in New Zealand domestic cats using a real-time PCR assay. J Feline Med Surg. 2013;15:1063-9.

14. Luria BJ, Levy JK, Lappin MR, Breitschwerdt EB, Legendre AM, Hernandez $J A$, et al. Prevalence of infectious diseases in feral cats in Northern Florida. J Feline Med Surg. 2004;6:287-96.

15. Willi B, Boretti FS, Meli ML, Bernasconi MV, Casati S, Hegglin D, et al. Real-time PCR investigation of potential vectors, reservoirs, and shedding patterns of feline hemotropic mycoplasmas. Appl Environ Microbiol. 2007:73:3798-802.
16. Sykes JE, Drazenovich NL, Ball LM, Leutenegger CM. Use of conventional and real-time polymerase chain reaction to determine the epidemiology of hemoplasma infections in anemic and nonanemic cats. J Vet Internal Med. 2007;21:685-93.

17. Tasker S, Helps CR, Day MJ, Gruffydd-Jones TJ, Harbour DA. Use of RealTime PCR to detect and quantify Mycoplasma haemofelis and 'Candidatus Mycoplasma haemominutum' DNA. J Clin Microbiol. 2003:41:439-41.

18. Flagstad A, Larsen S. The occurence of feline infectious anaemia in Denmark. Nord Vet Med. 1974:21:129-41.

19. Peters IR, Helps CR, Willi B, Hofmann-Lehmann R, Tasker S. The prevalence of three species of feline haemoplasmas in samples submitted to a diagnostics service as determined by three novel real-time duplex PCR assays. Vet Microbiol. 2008;126:142-50.

20. Willi B, Boretti FS, Tasker S, Meli ML, Wengi N, Reusch CE, Lutz H, Hofmann-Lehmann R. From Haemobartonella to hemoplasma: molecular methods provide new insights. Vet Microbiol. 2007;125:197-209.

21. Tasker S, Binns SH, Day MJ, Gruffydd-Jones TJ, Harbour DA, Helps CR, et al Use of a PCR assay to assess the prevalence and risk factors for Mycoplasma haemofelis and 'Candidatus Mycoplasma haemominutum' in cats in the United Kingdom. Vet Rec. 2003:152:193-8.

22. Messick JB. New perspectives about Hemotrophic mycoplasma (formerly, Haemobartonella and Eperythrozoon species) infections in dogs and cats. Vet Clin North Am Small Anim Pract. 2003;33:1453-65.

23. Lappin MR, Griffin B, Brunt J, Riley A, Burney D, Hawley J, et al. Prevalence of Bartonella species, haemoplasma species, Ehrlichia species, Anaplasma phagocytophilum, and Neorickettsia risticii DNA in the blood of cats and their fleas in the United States. J Feline Med Surg. 2006;8:85-90.

24. Woods JE, Brewer MM, Hawley JR, Wisnewski N, Lappin MR. Evaluation of experimental transmission of Candidatus Mycoplasma haemominutum and Mycoplasma haemofelis by Ctenocephalides felis to cats. Am J Vet Res. 2005:66:1008-12

25. Tasker S. Haemotropic mycoplasmas: what's their real significance in cats? J Feline Med Surg. 2010;12:369-81.

26. Bauer N, Balzer HJ, Thüre S, Moritz A. Prevalence of feline haemotropic mycoplasmas in convenience samples of cats in Germany. J Feline Med Surg. 2008;10:252-8.

27. Gentilini F, Novacco M, Turba ME, Willi B, Bacci ML, Hofmann-Lehmann R. Use of combined conventional and real-time PCR to determine the epidemiology of feline haemoplasma infections in northern Italy. J Feline Med Surg. 2009:11:277-85.

28. De Morais HA, Guimarães AM, Vidotto O, Baumann A, Biondo AW Messick Jb. Co-infection with Mycoplasma haemofelis and 'Candidatus Mycoplasma haemominutum' in three cats from Brazil. J Feline Med Surg. 2007;9:518-20.

29. Duarte A, Marques V, Correia JHD, Neto I, Brás BS, Rodrigues C, et al. Molecular detection of haemotropic Mycoplasma species in urban and rural cats from Portugal. J Feline Med Surg. 2015;17:516-22.

\section{Submit your next manuscript to BioMed Central and we will help you at every step:}

- We accept pre-submission inquiries

- Our selector tool helps you to find the most relevant journal

- We provide round the clock customer support

- Convenient online submission

- Thorough peer review

- Inclusion in PubMed and all major indexing services

- Maximum visibility for your research

Submit your manuscript at www.biomedcentral com/submit
BioMed Central 